๑) Open Access Full Text Article

\title{
Severity of Depressive Symptoms is Associated with Venous Thromboembolism in Hospitalized Patients with a Major Depressive Episode
}

\author{
Kazuhisa Yoshizawa (iD) \\ Masahiro Takeshima (iD ${ }^{2}$ \\ Sayaka Ishino ${ }^{3}$ \\ Masaya Ogasawara ${ }^{2}$ \\ Dai Fujiwara ${ }^{2}$ \\ Yu Itoh (iD) ${ }^{2}$ \\ Aya Imanishi ${ }^{2}$ \\ Hidenobu Ohta ${ }^{2}$ \\ Kazuo Mishima (iD ${ }^{2}$ \\ 'Department of Psychiatry, Akita \\ Prefectural Center for Rehabilitation and \\ Psychiatric Medicine, Daisen City, Akita, \\ 019-2492, Japan; ${ }^{2}$ Department of \\ Neuropsychiatry, Akita University \\ Graduate School of Medicine, Akita City, \\ Akita, 0I 0-8543, Japan; ${ }^{3}$ Department of \\ Neuropsychiatry, Sugawara Hospital, \\ Yurihonjo City, Akita, 015-0012, Japan
}

Correspondence: Masahiro Takeshima Department of Neuropsychiatry, Akita University Graduate School of Medicine, I-I-I, Hondo, Akita City, Akita, 0I0-8543, Japan

Tel +8I- |8-884-6|22

Fax +8I-18-884-6445

Email m.takeshima@med.akita-u.ac.jp
Purpose: A major depressive episode is a risk factor for venous thromboembolism (VTE) in psychiatric inpatients. However, it is unclear whether the severity of depressive symptoms or duration of the current depressive episode is associated with VTE. Further, the VTE prevalence among hospitalized patients with a major depressive episode receiving electroconvulsive therapy is unknown. This retrospective study examined factors associated with VTE among hospitalized patients with a major depressive episode and estimated the prevalence of VTE in such patients who underwent electroconvulsive therapy.

Patients and Methods: Patients with a major depressive episode hospitalized in the Department of Neuropsychiatry at Akita University Hospital between January 2018 and December 2020 were included. Data from the first week of hospitalization were extracted from medical records. VTE was diagnosed based on the findings of computed tomography. To evaluate whether the severity of depressive symptoms or duration of the current depressive episode was associated with VTE, logistic regression analysis was conducted after adjusting for covariates with known VTE risk factors (antidepressants, antipsychotics, and physical comorbidities).

Results: We analyzed 133 patients; of these, 14 were diagnosed with asymptomatic VTE. The severity of depressive symptoms (odds ratio: 1.220 , 95\% confidence interval: 1.081 $1.377, \mathrm{p}=0.001$ ) was significantly associated with VTE. The prevalence of VTE among those receiving electroconvulsive therapy was $35 \%(7 / 20)$.

Conclusion: The prevalence of VTE was $35 \%$ among patients receiving in-hospital electroconvulsive therapy for a major depressive episode. VTE should be considered for hospitalized patients with severe depressive symptoms and patients receiving in-hospital electroconvulsive therapy for a major depressive episode.

Keywords: bipolar depression, depressive symptoms, electroconvulsive therapy, unipolar depression, venous thromboembolism

\section{Introduction}

Venous thromboembolism (VTE) is a disease that includes deep vein thrombosis (DVT) and pulmonary embolism (PE). Numerous studies have reported that VTE is associated with sudden death, ${ }^{1}$ increased mortality, ${ }^{2}$ hospital costs, ${ }^{3,4}$ and longer hospital stays. ${ }^{3,4}$ In studies that examined causes of sudden death among patients with psychiatric disorders, PE accounted for 4-11\% of sudden deaths. ${ }^{5,6}$ Thus, identifying patient groups at a high risk of VTE is essential for developing strategies for prevention, early detection, and appropriate intervention. Psychiatric disorders, such as major depressive disorder ${ }^{7,8}$ and bipolar disorder, ${ }^{8}$ and therapeutic agents used for their 
treatment, such as antidepressants ${ }^{7}$ and antipsychotics, ${ }^{9,10}$ have been reported to be psychiatric risk factors for VTE. A recent study reported that $4.9 \%$ (258/5268) of patients hospitalized for psychiatric disorders had VTE, and that major depressive episodes were a risk factor for VTE. ${ }^{11}$ Therefore, more attention should be paid to VTE in patients hospitalized for a major depressive episode. However, it is unclear whether the severity of depressive symptoms and duration of the current depressive episode is associated with VTE because previous studies have not assessed these relationships. $^{11}$

Electroconvulsive therapy (ECT) is the most effective treatment for mood disorders, ${ }^{12}$ and often used for patients with severe or drug-resistant mood disorders in clinical practice. $^{12}$ Given that hospitalized patients with a major depressive episode receiving ECT generally have more severe depressive symptoms or longer duration of current depressive episodes than those not receiving ECT, patients receiving ECT may be at a higher risk for VTE. Further, a previous review concluded that ECT should be discontinued until thrombus disappearance when proximal DVT is diagnosed before or during an ECT session because of safety concerns. ${ }^{13}$ Although VTE risk assessment is recommended prior to $\mathrm{ECT},{ }^{14}$ no systematic study has examined the risk of VTE in patients with mood disorders undergoing ECT.

Our research group has been investigating VTE in patients hospitalized in psychiatric hospitals. Among the patients admitted to the psychiatry department of our hospital over an 8-year period, 2.3\% were diagnosed with VTE during their hospitalization, and about half of the VTE patients had a major depressive episode. ${ }^{15}$ We therefore focused on patients admitted for major depressive episodes and found VTE in $8.5 \%$ of patients hospitalized over 1.5 years from January 2018 to June $2019 .{ }^{16} \mathrm{We}$ hypothesized that more severe depressive symptoms and longer depressive episodes would be associated with the presence of VTE among patients hospitalized for a major depressive episode, and we explored these associations in previous studies, but failed to demonstrate an association. However, our previous study lacked power due to its small sample size and did not consider confounding factors. In addition, we hypothesized that patients with a major depressive episode receiving in-hospital ECT would have a higher prevalence of VTE because they often have more severe depressive symptoms or longer depressive episodes due to treatment resistance. ${ }^{12}$ Therefore, we conducted a retrospective chart review that doubled the duration of our previous study to test these hypotheses.

\section{Materials and Methods Study Participants}

This retrospective study included consecutive inpatients diagnosed with major depressive disorder (MDD), bipolar I disorder, or bipolar II disorder manifesting in a depressive state who underwent VTE screening within 1 week of hospitalization at the Department of Neuropsychiatry of Akita University Hospital over a 3-year period between January 1, 2018 and December 31, 2020. Patients were diagnosed according to the Diagnostic and Statistical Manual of Mental Disorders, Fifth Edition (DSM-5). The Department of Neuropsychiatry of Akita University Hospital is an acute psychiatric unit with 36 beds. In 2020, the average length of stay at the department was 28.2 days. Our hospital recommends VTE screening with D-dimer early after admission. In cases with a positive D-dimer result, our hospital also recommends VTE diagnosis with contrast computed tomography (CT), because very few doctors and technicians can perform ultrasonography compared to patients needing a VTE diagnosis. If there are no contraindications to contrast agents, contrast $\mathrm{CT}$ is performed based on both the clinician's harm-benefit analysis and the patient's written informed consent. Patients with a positive D-dimer result but no contrast $\mathrm{CT}$ results were excluded.

\section{Data Collection}

The following data were extracted from medical records: demographics (age and sex), type of treatment during hospitalization (ECT or pharmacotherapy), known and suspected risk factors for VTE, results of VTE screening with D-dimer, whether contrast CT was performed in patients with a positive D-dimer result, and presence or absence of VTE within 1 week of hospitalization.

\section{Psychiatric Risk Factors for VTE}

The following psychiatric risk factors for VTE were extracted: type of mood disorders based on the DSM-5 (MDD or bipolar disorder), ${ }^{7,8,11}$ catatonia based on DSM-5, ${ }^{11}$ use of antidepressants, ${ }^{7}$ and use of antipsychotics., ${ }^{9,10}$ Suspected risk factors for VTE were also extracted: severity of depression (total score on the 17-item Structured Interview Guide for the Hamilton Depression Rating Scale [HAM-D17]) ${ }^{17,18}$ and duration of the current depressive episode (weeks). The Japanese version of the 17-item Structured Interview Guide 
for the Hamilton Depression Rating Scale had sufficient reliability (In the analysis of variance test, the intraclass correlation coefficient of inter-rater reliability for the total score was 0.94 and internal consistency using Cronbach's alpha was 0.84) and concurrent validity (Kendall's $\tau$ B rank correlation between SIGH-D and BDI was $0.57(\mathrm{p}=0.04)){ }^{18}$

\section{Non-Psychiatric Risk Factors for VTE}

The following non-psychiatric risk factors for VTE were extracted: obesity (body mass index $[\mathrm{BMI}]\left[\mathrm{kg} / \mathrm{m}^{2}\right]$ ), ${ }^{19}$ trauma within 4 weeks, ${ }^{19}$ surgery within 4 weeks, ${ }^{19}$ malignancy, ${ }^{19}$ hypertension, ${ }^{19}$ hyperlipidemia, ${ }^{19}$ diabetes mellitus, ${ }^{19}$ pregnancy, ${ }^{19}$ oral contraceptive use, ${ }^{20}$ hormone replacement therapy, ${ }^{19}$ and history of VTE. ${ }^{19}$

\section{Other Factors Associated with VTE}

The following data associated with VTE were extracted: use of anticoagulants, D-dimer level $(\mu \mathrm{g} / \mathrm{mL})$, type of VTE (DVT without PE, PE without DVT, or DVT with PE), type of DVT (proximal or distal type), and symptomatic or asymptomatic VTE. Proximal DVT was defined as a thrombus in the iliac, femoral, or popliteal veins; distal DVT was defined as a thrombus in the anterior tibial vein, posterior tibial vein, or peroneal vein.

\section{VTE Screening with D-Dimer}

The plasma level of D-dimer was measured with the commercially available NANOPIA ${ }^{\circledR}$ latex agglutination assay kit (Sekisui Medical Company Limited, Tokyo, Japan), based on the same method commonly used for D-dimer assays such as the STA-Liatest D-dimer (Diagnostica Stago, Asnières, France), which contains latex microparticles coated with a monoclonal antibody specific for fragment $\mathrm{D}-\mathrm{D}$, in combination with an automated coagulation analyzer, the COAPRESTA 2000 (Sekisui Medical Co., Ltd., Tokyo, Japan). ${ }^{21,22}$ Concentrations were expressed in micrograms per milliliter $(\mu \mathrm{g} / \mathrm{mL})$, with a D-dimer level $\geq 1.0 \mu \mathrm{g} / \mathrm{mL}$ representing a positive result. With a cut-off value of $1.0 \mu \mathrm{g} / \mathrm{mL}$, the sensitivity of the NANOPIA kit for diagnosing VTE was $99.6 \%{ }^{22}$

\section{CT Imaging}

In cases with a positive D-dimer result $(\geq 1.0 \mu \mathrm{g} / \mathrm{mL})$, contrast CT was performed to reach a diagnosis within $12 \mathrm{~h}$ after measurement, if the patient had no contraindications to contrast agents; iodine or iodine contrast agent sensitivity; severe thyroid disease; bronchial asthma; severe renal dysfunction; severe liver dysfunction; severe heart failure; macroglobulinemia; multiple myeloma; tetany; or pheochromocytoma. VTE (PE or DVT) was detected using multidetector-row helical CT scanning on two different devices (Discovery CT750HD and Discovery CT750HD-A; GE Healthcare Japan, Tokyo, Japan), with a slice thickness of $1.25 \mathrm{~mm}$ at 20 seconds (early phase) for detecting pulmonary arterial embolization and at 210 seconds (delayed phase) for detecting DVT. Low-osmolar iodinated contrast medium (Omnipaque 300; Daiichi Sankyo Company Limited, Tokyo, Japan) was intravenously injected at a rate of $4.0 \mathrm{~mL} / \mathrm{second}$ using a power injector, up to a total amount of body weight $(\mathrm{kg}) \times 2+30$ $(\mathrm{mL})$ or a maximum of $150 \mathrm{~mL}$. Diagnostic radiology specialists of the Japan Radiological Society or doctors with more than a decade's experience in radiological diagnosis analyzed the CT scans. The physicians assessing the CT scans were not blinded to the D-dimer results. Contrast CT had comparable performance in the diagnosis and exclusion of DVT with a $95 \%$ concordance compared to ultrasound. $^{23}$

\section{Statistical Analysis}

Statistical analyses were performed with SPSS Statistics 27.0 (IBM Corp., Armonk, NY, USA). Summary measurements are presented as the median $(25 \%-75 \%$ percentile $)$. The Mann-Whitney $U$-test was used to compare mean values, and Fisher's exact test was used to compare sex ratio and other variables between the VTE-positive group and the VTE-negative group. To evaluate whether the severity of depressive symptoms and duration of the current depressive episode were associated with VTE, binary logistic regression analysis by the forced entry method was conducted after adjusting for the following covariates: age, sex, BMI, type of mood disorders (MDD or bipolar disorder), catatonia, use of antidepressants, use of antipsychotics, total score on the HAM-D17, duration of the current depressive episode, trauma within 4 weeks, surgery within 4 weeks, malignancy, hypertension, hyperlipidemia, diabetes mellitus, pregnancy, oral contraceptive use, hormone replacement therapy, history of VTE, and use of anticoagulants. ECT was not included in the logistic regression analysis as a covariate because ECT was suspected to be multicollinear with the severity of depressive symptoms or the duration of the current depressive episodes. If there was a nominal variable with no patients in the VTE-positive or VTE-negative group, the variables were not included in the logistic regression analysis. 


\section{Ethical Procedures}

This study was approved by the Ethical Committee for Human Research of Akita University (no. 2596) and adhered to the ethical guidelines of clinical and epidemiological research. This retrospective study was exempt from the requirement of informed consent, as it involved de-identified data acquired during routine care of patients. This study was conducted in accordance with the Declaration of Helsinki.

\section{Results}

\section{Prevalence of VTE Among Hospitalized Patients with Unipolar or Bipolar Depression}

During the study period, 154 adult Japanese patients were hospitalized to undergo treatment for a major depressive episode at our hospital. No patients were undergoing acute treatment for VTE. After excluding duplicate cases and one case undergoing acute treatment for arterial dissection, 141 patients were identified. Almost all patients $(n=139)$ underwent VTE screening, but two patients did not undergo the screening within the first week of hospitalization. None of the patients had contraindications to iodinated contrast medium, had used oral contraceptives, or had received hormone replacement therapy or surgery within the previous 4 weeks. Eleven patients were taking anticoagulants (8, atrial fibrillation; 2, prevention for ischemic stroke; and 1, prevention for VTE). No patient was pregnant. Thirty-six patients were screened positive for VTE. Thirty patients then underwent contrast CT, but six patients did not agree to the procedure. Finally, 133 patients were analyzed in this study (91 women, median age: $64[47,75]$ years). The level of D-dimer of those six patients ranged from 1.05 to $3.80 \mu \mathrm{g} / \mathrm{mL}$, with an average of $2.10 \mu \mathrm{g} / \mathrm{mL}$. Among the 133 patients analyzed in this study, $10.5 \%$ (14/133) were diagnosed with asymptomatic VTE (7 with PE only, 4 with DVT only, and 3 with both PE and DVT). All patients diagnosed with DVT had proximal DVT. All patients with VTE improved on anticoagulant therapy without showing any side effects such as bleeding. Figure 1 shows the scatter plot of the severity of depressive symptoms and duration of the current depressive episode among inpatients with depression with or without VTE. The severity of depression, as measured by the HAMD-17, ranged from 8 to 43 points in the VTEpositive group and from 3 to 34 points in the VTEnegative group. The duration of the current depressive episode ranged from 2 to 350 weeks in the VTE-positive group and from 7 to 40 weeks in the VTE-negative group. Table 1 shows the demographic and clinical characteristics

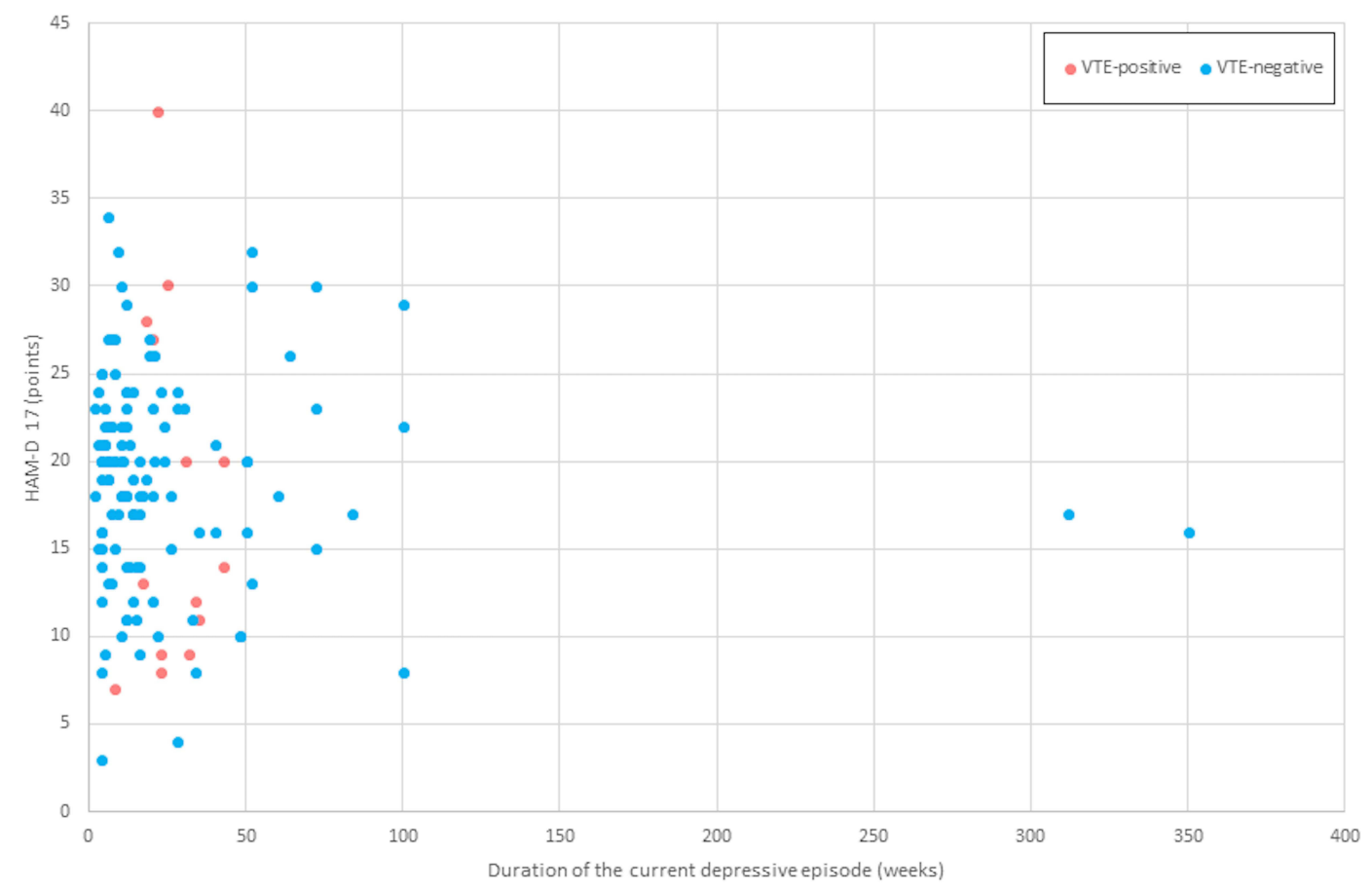

Figure I Scatter plot of the severity of depressive symptoms and duration of the current depressive episode among inpatients with depression with or without VTE. Note: VTE-positive is indicated by a red circle and VTE-negative by a blue circle.

Abbreviations: HAM-DI7, 17-item Hamilton Depression Rating Scale; VTE, venous thromboembolism. 
Table I Clinical and Demographic Characteristics of VTE-Positive and VTE-Negative Patients

\begin{tabular}{|c|c|c|c|}
\hline Characteristics & VTE-Positive $(n=14)$ & VTE-Negative $(\mathrm{n}=119)$ & $\mathbf{p}$ \\
\hline \multicolumn{4}{|l|}{ Demographics } \\
\hline Age (years) & 69 (60-79) & $62(46-75)$ & $0.09^{\mathrm{a}}$ \\
\hline Women & 9 & 82 & $0.77^{\mathrm{b}}$ \\
\hline BMI $\left(\mathrm{kg} / \mathrm{m}^{2}\right)$ & $20.15(18.15-24.05)$ & $21.5(19.5-24.6)$ & $0.26^{\mathrm{a}}$ \\
\hline \multicolumn{4}{|c|}{ Non-psychiatric risk factors for VTE } \\
\hline Trauma & I & 1 & $0.20^{\mathrm{b}}$ \\
\hline Malignancy & 3 & 7 & $0.07^{b}$ \\
\hline Hypertension & 4 & 37 & $1^{\mathrm{b}}$ \\
\hline Hyperlipidemia & 0 & 19 & $0.22^{\mathrm{b}}$ \\
\hline Diabetes mellitus & 0 & 17 & $0.21^{\mathrm{b}}$ \\
\hline \multicolumn{4}{|c|}{ Psychiatric risk factors for VTE } \\
\hline Bipolar depression & 3 & 22 & $0.73^{b}$ \\
\hline Catatonia & $\mathrm{I}$ & 0 & $0.11^{\mathrm{b}}$ \\
\hline Use of antidepressants & 9 & 90 & $0.35^{\mathrm{b}}$ \\
\hline Use of antipsychotics & 8 & 36 & $0.07^{b}$ \\
\hline \multicolumn{4}{|c|}{ Other factors associated with VTE } \\
\hline History of VTE & I & 4 & $0.43^{b}$ \\
\hline Use of anticoagulants & I & 10 & $\mathrm{I}^{\mathrm{b}}$ \\
\hline D-dimer $(\mu \mathrm{g} / \mathrm{mL})$ & $5.50(2.83-7.96)$ & $0.62(0.5 \mathrm{I}-0.87)$ & $<0.001^{\mathrm{a}, *}$ \\
\hline \multicolumn{4}{|c|}{ Suspected psychiatric risk factors for VTE } \\
\hline Duration (weeks) & $13.50(9.00-27.25)$ & $12(6-24)$ & $0.34^{\mathrm{a}}$ \\
\hline HAM-DI7 & $24.00(19.50-34.25)$ & $19.00(16-23)$ & $0.003^{\mathrm{a}, *}$ \\
\hline
\end{tabular}

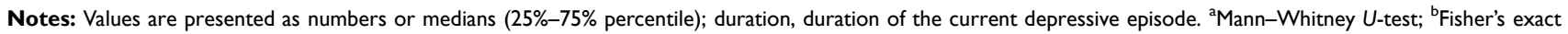
test; *Significant $p$-values $(\mathrm{p}<0.05)$.

Abbreviations: BMI, body mass index; HAM-DI7, I7-item Hamilton Depression Rating Scale; VTE, venous thromboembolism.

of the VTE-positive and VTE-negative groups. The VTEpositive group had significantly worse severity of depressive symptoms than the VTE-negative group, but there were no significant differences between the two groups in the other variables.

\section{Prevalence of VTE Among ECT-Treated Patients}

During the study period, 20 patients received in-hospital ECT. The mean age was 61.1 years. Among the 20 patients, nutritional intake was inadequate for 11 patients, and five were administered intravenous fluids to compensate for the inadequate nutritional intake. Seven patients who received in-hospital ECT were diagnosed with asymptomatic VTE (five with PE only, one with DVT only, and one with both PE and DVT). All seven patients diagnosed with VTE prior to ECT were treated with direct oral anticoagulants. ECT was administered in two patients after thrombus resolution on contrast-enhanced $\mathrm{CT}$ and in five patients after normalization of D-dimer level. Initiation of ECT was based on the clinical decision of the cardiologist, anesthesiologist, and psychiatrist. ECT initiation was delayed by an average of 11 days (range: 0-21 days) due to VTE treatment.

\section{Logistic Regression Analysis}

To evaluate whether the severity of depressive symptoms was associated with VTE, a logistic regression analysis was performed after adjusting for the covariates other than catatonia because no patients with catatonia were present in the VTE-negative group. Table 2 shows the results of logistic regression analysis. Severity of depressive symptoms (odds ratio: 1.220, 95\% confidence interval: $1.081-1.377, \mathrm{p}=0.001$ ) was significantly associated with VTE. 
Table 2 Logistic Regression Analysis of Factors Associated with VTE

\begin{tabular}{|c|c|c|c|c|c|}
\hline Variables & B (SE) & Wald & Odds Ratio & $95 \% \mathrm{Cl}$ & p-value \\
\hline Age & $0.044(0.031)$ & 1.977 & 1.045 & 0.983 to $\mathrm{I} . \mathrm{III}$ & 0.160 \\
\hline Sex & $-0.257(0.78 I)$ & 0.109 & 0.773 & 0.167 to $3.57 \mathrm{I}$ & 0.742 \\
\hline BMI & $-0.025(0.094)$ & 0.074 & 0.975 & 0.812 to 1.171 & 0.785 \\
\hline Trauma & 2.799 (1.797) & 2.427 & 16.432 & 0.485 to 556.138 & 0.119 \\
\hline Malignancy & 1.267 (I.023) & 1.534 & 3.550 & 0.478 to 26.348 & 0.215 \\
\hline Hypertension & $0.278(0.869)$ & 0.102 & 1.320 & $0.24 I$ to 7.244 & 0.749 \\
\hline Bipolar depression & $-0.032(1.187)$ & 0.001 & 0.969 & 0.095 to 9.926 & 0.979 \\
\hline Antidepressants & $-0.474(0.875)$ & 0.294 & 0.622 & 0.112 to 3.457 & 0.588 \\
\hline Antipsychotics & $1.656(0.886)$ & 3.492 & 5.237 & 0.922 to 29.739 & 0.062 \\
\hline History of VTE & 1.008 (I.432) & 0.495 & $2.74 I$ & 0.165 to 45.410 & 0.482 \\
\hline Anticoagulants & $0.213(1.418)$ & 0.023 & 1.238 & 0.077 to 19.926 & 0.880 \\
\hline Duration & $-0.002(0.011)$ & 0.024 & 0.998 & 0.977 to 1.020 & 0.878 \\
\hline HAM-DI7 & $0.199(0.062)$ & 10.316 & 1.220 & 1.081 to 1.377 & $0.001 *$ \\
\hline
\end{tabular}

Note: *Significant $p$-value $(p<0.05)$.

Abbreviations: BMI, body mass index; Cl, confidence interval; HAM-DI7, I7-item Hamilton Depression Rating Scale; SE, standard error; VTE, venous thromboembolism.

\section{Discussion}

This study had two main findings. First, severity of depressive symptoms, but not duration of the current depressive episode, was associated with VTE in hospitalized patients with a major depressive episode. Second, the prevalence of VTE in patients with a major depressive episode receiving in-hospital ECT was as high as $35 \%$. These results support our hypothesis that more severe depressive symptoms are associated with the presence of VTE among patients hospitalized for a major depressive episode, and patients with a major depressive episode receiving in-hospital ECT have a higher prevalence of VTE, while refuting the hypothesis that longer depressive episodes would be associated with the presence of VTE among patients hospitalized for a major depressive episode.

To our knowledge, this is the first study to examine the association between VTE and severity of depressive symptoms among inpatients with a major depressive episode considering confounding factors. This study could not conclude whether the severity of depressive symptoms is a risk factor for VTE because the study observed cross-sectional findings rather than causal relationships. However, asymptomatic VTE is generally unlikely to cause depressive symptoms; therefore, the severity of depressive symptoms, or some factor related to depressive symptoms, may cause VTE among inpatients with a major depressive episode. In fact, a previous large, prospective, population-based study has reported that patients who feel depressed often are 1.59-fold more likely to develop VTE than those who do not. ${ }^{24}$
Another longitudinal study has reported that patients with VTE who have higher levels of depressive symptoms have a greater risk of recurrent VTE. ${ }^{25}$ Therefore, depressive symptoms may be associated with VTE. However, when interpreting the results of this study, the followings should be considered. First, there was a significant superposition in the percentile intervals for the VTE-positive and negative groups regarding depression severity. In addition, when this association was analyzed in the logistic regression, the confidence interval for the odds ratio was 1.081 to 1.377 , suggesting that available data are compatible with both very large and very small effects. This uncertainty in the effect estimates, combined with the known volatility of effect size estimates based on the small samples (and there were very few VTE-positive patients) highlights the level of caution required when interpreting these results, compared to p-values alone. Further, since the sample size of this study was not sufficient, we did not investigate which sub-items of the HAM-D17 were associated with VTE. Further research is warranted to investigate the risk factors for VTE in patients with major depressive episode. Second, "the severity of depressive symptoms" might indirectly be a positive predictor due to "an increased dose of antidepressants and antipsychotics" in patients with a major depressive episode. In this study, the presence or absence of antidepressant or antipsychotic medication was considered as a covariate, but the dose was not. Hence, the dosage of antidepressants and antipsychotics should be considered in future studies that 
investigate factors associated with VTE among patients with a major depressive episode.

We also hypothesized that the longer the duration of the depressive episodes, the higher the prevalence of VTE. However, our hypothesis was not supported by the data, suggesting that even a short depressive episode could lead to VTE.

Approximately one-third of the patients with a major depressive episode undergoing ECT had asymptomatic VTE (5 with PE only, 1 with DVT only, and 1 with both PE and DVT). A previous study that examined DVT using ultrasonography reported that $25.3 \%$ of hospitalized catatonia patients had $\mathrm{DVT}^{26}$ and a previous study that examined VTE using contrast-enhanced CT reported that $61.1 \%$ had VTE. ${ }^{15}$ These results suggested that hospitalized patients with a major depressive episode undergoing ECT should be paid as much attention for VTE as patients with catatonia. Although previous case studies reported symptomatic PE early after ECT administration, ${ }^{27,28}$ whether screening for VTE should be mandatory in all patients undergoing ECT is controversial because it is unclear whether ECT increases the incidence of serious adverse events including symptomatic PE during and after ECT in patients with asymptomatic VTE. Given the insufficient evidence, the decision to screen for VTE should be based on an individual patient's VTE risk and physical problems. When VTE is diagnosed before or during an ECT session, clinicians should carefully decide whether to perform or wait for ECT after comprehensively considering the severity of psychiatric symptoms, physical condition, clot size, bleeding risk, etc. because there is no consensus on whether ECT should be administered after the blood clots have completely disappeared by anticoagulant therapy or early after the start of anticoagulant therapy. A recent review on patients with schizophrenia and catatonia has concluded that ECT can be safely administered in patients with anticoagulant-treated distal DVT, but not in patients with proximal DVT (not until the thrombus disappears). ${ }^{13}$ Although no serious adverse events have been reported in patients receiving ECT under anticoagulant therapy in previous studies, the safety of anticoagulant therapy for patients undergoing ECT has not been established because all previous studies were small case series. ${ }^{29,30}$ Further well-designed studies are warranted to examine the safety of anticoagulants for patients undergoing ECT and the benefits of early initiation of ECT.

\section{Limitations}

This study has several limitations. First, the sample size is too small to generate true positive significant predictors for logistic regression analysis. Considering that the event of interest was only 14/133 and the study had 14 predict variables, a sample size of 133 was too small and would lead to false positive results and overfitting. Moreover, a lack of statistical significance on the presence of malignancy or past history of VTE as a risk factor for VTE is likely to represent a type II error resulting from the small sample size in this study. Further studies with a larger sample size are warranted to confirm the results of this study. Second, this study may have a selection bias because VTE was not investigated in all patients, even upon positive D-dimer test results during VTE screening. Third, the study participants were patients hospitalized with a major depressive episode, not outpatients. Therefore, it is unclear whether the severity of depressive symptoms is associated with VTE in outpatients. Fourth, unequivocal risk factors for developing VTE such as hypercoagulable status, immobility, and dehydration were not considered in this study. ${ }^{19}$

Special attention should be paid to VTE in hospitalized patients with severe depressive symptoms, regardless of whether they receive ECT. Nevertheless, VTE should be considered in patients receiving in-hospital electroconvulsive therapy for a major depressive episode.

\section{Conclusion}

The prevalence of VTE was high (35\%) among patients receiving in-hospital electroconvulsive therapy for a major depressive episode. The severity of depressive symptoms, but not the duration of depressive episodes, was associated with VTE in hospitalized patients with a major depressive episode.

\section{Data Sharing Statement}

The datasets used and analyzed during the current study are available from the corresponding author on reasonable request.

\section{Role of the Funding Source}

This research did not receive any specific grant from funding agencies in the public, commercial, or not-forprofit sectors. 


\section{Acknowledgments}

We would like to thank Editage for English language editing.

\section{Author Contributions}

All authors made a significant contribution to the work reported, whether that is in the conception, study design, execution, acquisition of data, analysis and interpretation, or in all these areas; took part in drafting, revising or critically reviewing the article; gave final approval of the version to be published; have agreed on the journal to which the article has been submitted; and agree to be accountable for all aspects of the work.

\section{Disclosure}

The authors report no conflicts of interest in this work.

\section{References}

1. Ota M, Nakamura M, Yamada N, et al. Prognostic significance of early diagnosis in acute pulmonary thromboembolism with circulatory failure. Heart Vessels. 2002;17(1):7-11. doi:10.1007/ s003800200036

2. Søgaard KK, Schmidt M, Pedersen L, Horváth-Puhó E, Sørensen HT. 30-year mortality after venous thromboembolism. Circulation. 2014;130:829-836. doi:10.1161/CIRCULATIONA HA.114.009107

3. Amin A, Deitelzweig S, Bucior I, et al. Frequency of hospital readmissions for venous thromboembolism and associated hospital costs and length of stay among acute medically ill patients in the US. $J$ Med Econ. 2019;22:1119-1125. doi:10.1080/13696998.20 19.1618862

4. Amin A, Neuman WR, Lingohr-Smith M, Menges B, Lin J. Influence of the duration of hospital length of stay on frequency of prophylaxis and risk for venous thromboembolism among patients hospitalized for acute medical illnesses in the USA. Drugs Context. 2019;8:1-9. doi:10.7573/dic. 212568

5. Manu P, Kane JM, Correll CU. Sudden deaths in psychiatric patients. J Clin Psychiatry. 2011;72(7):936-941. doi:10.4088/JCP.10m0 6244gry

6. Maruyama J, Matsunaga R, Sato F, et al. Preventing Pulmonary Thromboembolism in Psychiatric Hospital. Tokyo: Nakayamashoten; 2009.

7. Kunutsor SK, Seidu S, Khunti K. Depression, antidepressant use, and risk of venous thromboembolism: systematic review and meta-analysis of published observational evidence. Ann Med. 2018;50:529-537. doi:10.1080/07853890.2018.1500703

8. Lin CE, Chung CH, Chen LF, Chien WC. Increased risk for venous thromboembolism among patients with concurrent depressive, bipolar, and schizophrenic disorders. Gen Hosp Psychiatry. 2019;61:34-40. doi:10.1016/j.genhosppsych.2019.10.003

9. Jonsson AK, Schill J, Olsson H, Spigset O, Hagg S. Venous thromboembolism during treatment with antipsychotics: a review of current evidence. CNS Drugs. 2018;32:47-64. doi:10.1007/s40263-0180495-7

10. Barbui C, Conti V, Cipriani A. Antipsychotic drug exposure and risk of venous thromboembolism: a systematic review and meta-analysis of observational studies. Drug Saf. 2014;37:79-90. doi:10.1007/ s40264-013-0127-6
11. Ishida T, Shibahashi K, Sugai S, et al. Development of a risk stratification scoring system for deep vein thrombosis upon psychiatric admission. J Psychosom Res. 2021;147:110540. doi:10.1016/j. jpsychores.2021.110540

12. Bahji A, Hawken ER, Sepehry AA, Cabrera CA, Vazquez G. ECT beyond unipolar major depression: systematic review and meta-analysis of electroconvulsive therapy in bipolar depression. Acta Psychiatr Scand. 2019;139:214-226.

13. Warriach ZI, Shamim SA, Saeed A, Kashif S, Malik BH. Association of the thrombo-embolic phenomenon with electroconvulsive therapy treatment in schizophrenia with catatonia patient. Cureus. 2019;11 (9):e5656.

14. Tess AV, Smetana GW. Medical evaluation of patients undergoing electroconvulsive therapy. $N$ Engl J Med. 2009;360:1437-1444. doi:10.1056/NEJMra0707755

15. Takeshima M, Ishikawa H, Shimizu K, Kanbayashi T, Shimizu T. Incidence of venous thromboembolism in psychiatric inpatients: a chart review. Neuropsychiatr Dis and Treat. 2018;14:1363-1370. doi:10.2147/NDT.S162760

16. Takeshima M, Ishikawa H, Umeta Y, et al. Prevalence of asymptomatic venous thromboembolism in depressive inpatients. Neuropsychiatr Dis Treat. 2020;16:579-587. doi:10.2147/NDT. $\mathrm{S} 243308$

17. Williams JB. A structured interview guide for the Hamilton Depression Rating Scale. Archiv Gen Psychiatry. 1988;45 (8):742-747. doi:10.1001/archpsyc.1988.01800320058007

18. Narita T, Kim N, Nakane Y, et al. Reliability and validity of structured interview guide for the Hamilton depression rating scale (SIGH-D). Jpn J Clin Psychopharmacol. 2003;6:77-82.

19. Goldhaber SZ. Risk factors for venous thromboembolism. J Am Coll Cardiol. 2010;56:1-7. doi:10.1016/j.jacc.2010.01.057

20. Baratloo A, Safari S, Rouhipour A, et al. The risk of venous thromboembolism with different generation of oral contraceptives; a systematic review and meta-analysis. Emergency. 2014;2:1.

21. Waser G, Kathriner S, Wuillemin WA. Performance of the automated and rapid STA Liatest D-dimer on the STA-R analyzer. Thromb Res. 2005;116:165-170. doi:10.1016/j.thromres.2004.12.003

22. Yamaki T, Nozaki M, Sakurai H, et al. Combined use of pretest clinical probability score and latex agglutination D-dimer testing for excluding acute deep vein thrombosis. $J$ Vasc Surg. 2009;50:1099-1105. doi:10.1016/j.jvs.2009.06.059

23. Goodman LR, Stein PD, Matta F, et al. CT venography and compression sonography are diagnostically equivalent: data from PIOPED II. AJR Am J Roentgenol. 2007;189(5):1071-1076. doi:10.2214/ AJR.07.2388

24. Enga KF, Braekkan SK, Hansen-Krone JJ, Hansen JB. Emotional states and future risk of venous thromboembolism: the Tromso Study. J Thromb Haemost. 2012;107:485-493. doi:10.1160/TH11-09-0667

25. Von Känel R, Margani A, Stauber S, et al. Depressive symptoms as a novel risk factor for recurrent venous thromboembolism: a longitudinal observational study in patients referred for thrombophilia investigation. PLoS One. 2015;10:e0125858. doi:10.1371/journal.pone. 0125858

26. Ishida T, Sakurai H, Watanabe K, Iwashita S, Mimura M, Uchida H. Incidence of deep vein thrombosis in catatonic patients: a chart review. Psychiatry Res. 2016;241:61-65. doi:10.1016/j. psychres.2016.04.105

27. Buday J, Albrecht J, Mares T, et al. A case report of pulmonary embolism during electroconvulsive therapy and its further application after somatic stabilization. Brain Stimul. 2020;13:250-252. doi:10.1016/j.brs.2019.09.004

28. Kursawe HK, Schmikaly R. Fatal pulmonary embolism following mitigated electroconvulsive therapy. A case report from the intensive care viewpoint. Psychiatr Neurol Med Psychol Beih. 1988;40:107-108. 
29. Hirata T, Yasuda K, Uemura T, et al. Electroconvulsive therapy while receiving oral anticoagulation for deep venous thrombosis: report on eight cases and a review of the literature. Psychosomatics. 2019;60:402-409. doi:10.1016/j.psym.2018.10.007
30. Mehta V, Mueller PS, Gonzalez-Arriaza HL, Pankratz VS, Rummans TA. Safety of electroconvulsive therapy in patients receiving long-term warfarin therapy. Mayo Clin Proc. 2004;79 (11):1396-1401. doi:10.4065/79.11.1396

\section{Publish your work in this journal}

Neuropsychiatric Disease and Treatment is an international, peerreviewed journal of clinical therapeutics and pharmacology focusing on concise rapid reporting of clinical or pre-clinical studies on a range of neuropsychiatric and neurological disorders. This journal is indexed on PubMed Central, the 'PsycINFO' database and CAS, and is the official journal of The International Neuropsychiatric Association (INA). The manuscript management system is completely online and includes a very quick and fair peer-review system, which is all easy to use. Visit http://www.dovepress.com/testimonials.php to read real quotes from published authors.

Submit your manuscript here: https://www.dovepress.com/neuropsychiatric-disease-and-treatment-journal 